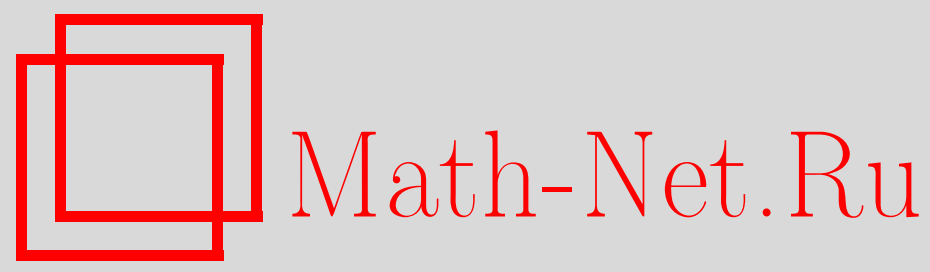

А. А. Панов, О числе периодических решений полиномиальных дифференциальных уравнений, Матем. заметки, 1998, том 64, выпуск 5, 720-727

DOI: https://doi.org/10.4213/mzm1448

Использование Общероссийского математического портала Math-Net.Ru подразумевает, что вы прочитали и согласны с пользовательским соглашением http://www.mathnet.ru/rus/agreement

Параметры загрузки:

IP: 54.92 .164 .108

26 апреля 2023 г., $11: 39: 13$

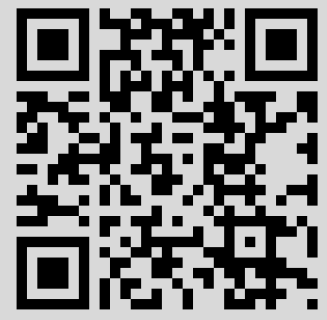




\section{О ЧИСЛЕ ПЕРИОДИЧЕСКИХ РЕШЕНИЙ ПОЛИНОМИАЛЬНЫХ ДИФФЕРЕНЦИАЛЬНЫХ УРАВНЕНИЙ}

\section{А. А. Панов}

Для специальных классов обыкновенных дифференциальных уравнений $n$-го порядка с переменньми коэффициентами получены оценки числа периодических решений.

Библиографияя: 5 названий.

Введение. В работе исследуется число периодических решений полиномиального дифференциального уравнения с периодическими коэффициентами вида

$$
\begin{gathered}
\dot{x}=x^{n}+a_{n-1}(t) x^{n-1}+\cdots+a_{0}(t), \\
a_{j}(t+T)=a_{j}(t) .
\end{gathered}
$$

Вопрос об оценке сверху числа решений уравнения (1) может считаться облегченным вариантом шестнадцатой проблемы Гильберта [1]. Однако и в этом варианте проблема решена лишь для $n \leqslant 3$. Для таких $n$ число предельных циклов уравнения (1) не превышает $n$. Для $n=1$ это утверждение следует из линейности уравнения. При $n=2$ преобразование монодромии уравнения (1) за период дробно-линейно, нетождественно и поэтому не может иметь больше двух неподвижных точек. При $n=3$ число периодических решений оценено в работах [2] и [3] (с указанием на то, что результат принадлежит Смейлу).

В п. 2 настоящей работы этот результат усилен. А именно, доказано, что уравнение

$$
\dot{x}=x^{2 n+1}+a(t) x^{2}+b(t)
$$

имеет не более трех периодических решений. Доказательство основано на монотонности по $t$ двойного отношения значений любых четырех решений уравнения (2).

При $n=4$ число периодических решений уравнения (1) может быть равно шести [2]. В п. 3 настоящей работы построен новьй класс уравнений (1) с $n=4$ и шестью периодическими решениями. Построение основано на существовании поля плоскостей в $\mathbb{R}^{3}$, свойства которого в значительной мере определяют поведение решений уравнения (1) при $n=4$.

При малых коэффициентах уравнение (1) имеет не более $n$ периодических решений с учетом кратности. Это следует из принципа Руше, примененного к комлексификации преобразования монодромии за период $T$. В п. 1 явно указана зависящая от $T$ и $n$ константа $C$ такая, что неравенства $\left|a_{j}\right|<C$ гарантируют наличие не более чем $n$ циклов у уравнения (1).

Работа вьполненапри подлержке фонда CRDF, грант RM1-229, фонда INTAS, грант № 93-05-07, и Российского фонда фундаментальных исследований, грант № 95-01-01258. 
1. Уравнения с малыми периодическими коэффициентами. Рассмотрим уравнение вида (1) с периодически зависящими от времени коэффициентами $a_{i}(t+T)=$ $a_{i}(t)$. Пусть $\varphi(x, t)$ - решение этого уравнения с начальным условием $\varphi(x, 0)=x . \mathrm{B}$ дальнейшем через $\Phi$ мы будем обозначать отображение последования $\Phi: x \rightarrow \varphi(x, T)$.

ТЕОРЕМА 1. Пусть $T=1 /(6 n)$ и для любого $t$ корни многочлена

$$
x^{n}+a_{n-1}(t) x^{n-1}+\cdots+a_{0}(t),
$$

включая комплексные, по модулю не превосходят $\pi /(2 n)$. Тогда отобрахсение последования имеет не более $n$ неподвижных точек.

ЗАмЕчаниЕ. Можно было бы вместо периода $T=1 /(6 n)$ взять период $T=2 \pi$ и тогда константу $\pi /(2 n)$ в условии теоремы следовало бы заменить на

$$
\frac{\pi / 12}{(12 \pi n)^{1 /(n-1)}} .
$$

Для доказательства теоремы комплексифицируем уравнение (1) по $x$ и разложим правую часть на множители:

$$
\dot{z}=\left(z-b_{1}(t)\right)\left(z-b_{2}(t)\right) \cdots\left(z-b_{n}(t)\right), \quad\left|b_{i}(t)\right|<\frac{\pi}{2 n} .
$$

Предполагается, что все функции $b_{i}(t)$ гладкие. Будет доказано, что отображение последования

$$
\Phi: \mathbb{C} \rightarrow \mathbb{C}, \quad z \mapsto \varphi\left(z, \frac{1}{6 n}\right),
$$

имеет ровно $n$ неподвижных точек. Доказательство опирается на следующие две леммы.

ЛЕмма 1. Фазовая кривая любого периодического решения с периодом $T$ уравнения (3) обязана пересекаться с кругом $|z| \leqslant 1$.

ЛЕмма 2. Существует число $R(n), R(n)>1$, удовлетворяющее следуюшим двум условиям:

1) для всякого уравнения вида (3) отображение последования Ф определено на всем круге $|z| \leqslant R(n)$;

2) ни одна из периодических фазовых кривых уравнения (3) не пересекает окружность $|z|=R(n)$.

Покажем, как из этих двух лемм следует теорема.

ДоКАЗАТЕЛЬСТво тЕОРЕмЫ. Из лемм 1 и 2 вытекает, что все периодические фазовые кривые уравнения (3) лежат внутри круга $|z|<R(n)$. Другими словами, для всякого уравнения вида (3) отображение $z \mapsto \Phi(z)-z$ не обращается в 0 на окружности $|z|=R(n)$. Поэтому можно определить индекс образа этой окружности относительно точки 0 под действием отображения последования $\Phi$.

По непрерьвности для всех уравнений вида (3) этот индекс один и тот же, а именно, он равен $n$. По теореме Руше он в точности равен числу неподвижных точек отображения последования $\Phi$, т.е. числу периодических решений. Теорема доказана.

Приступим теперь к доказательству лемм. 
ДоКАЗАТЕЛЬСтво ЛЕмМы 1. Разобьем область $|z|>1$ выходящими из нуля лучами на $2 n-2$ равных сектора так, чтобы положительная действительная полуось была биссектрисой первого сектора. Пронумеруем эти секторы против часовой стрелки числами от 1 до $2 n-2$. Те секторы, которым соответствуют четные номера, назовем четны.ми, остальные - нечетным.и.

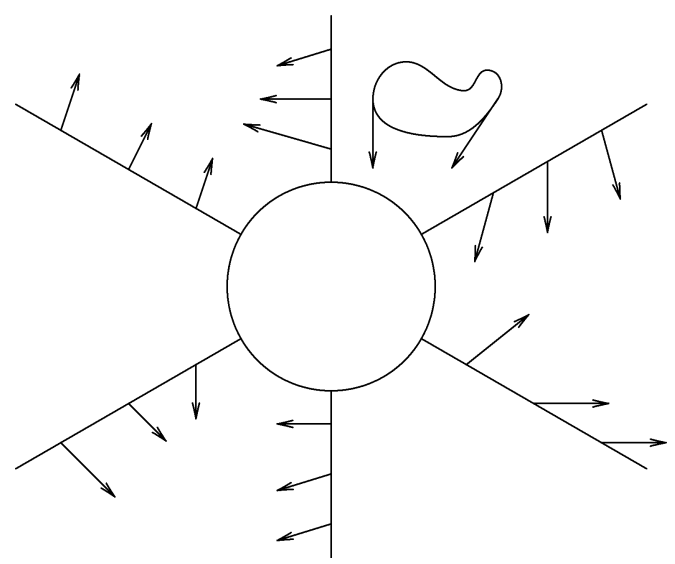

Рис. 1

Легко показать, что в любой момент времени векторное поле (3) на каждом из $2 n-2$ лучей смотрит в нечетную область (рис. 1). Кроме того, в четной области не существует ни одной точки, в которой вектор направлен точно в 0 , а в нечетной области не существует точки, в которой вектор направлен строго от 0 .

Допустим теперь, что у уравнения (3) существует периодическое решение, фазовая кривая которого лежит вне круга $|z| \leqslant 1$. Из сказанного вьше ясно, что такая фазовая кривая должна целиком лежать в одном из секторов (если она вошла в нечетньй сектор, то там и останется, см. рис. 1). На замкнутой фазовой кривой, лежащей в одном секторе, должны найтись две точки, в одной из которых вектор поля должен быть направлен в 0 , а в другой - от 0 (рис. 1), но такого быть не может. Лемма доказана.

ДокАЗАТЕЛЬСТво ЛЕмМЫ 2. Для доказательства достаточно указать число $R(n)$ такое, что точка, стартующая с окружности $|z|=R(n)$, за период $T=1 /(6 n)$, во-первых, не успеет достигнуть единичного круга $|z| \leqslant 1$ и вернуться обратно и, во-вторых, не уйдет на бесконечность. Оценим снизу время $t_{1}$, за которое точка, стартующая с окружности $|z|=R(n)$, сможет достигнуть единичного круга $|z| \leqslant 1$, и время $t_{2}$, за которое точка, стартующая с окружности $|z|=R(n)$, уйдет на бесконечность. Воспользуемся тем, что радиальная скорость на решении, проходящем через точку $z$, не превосходит $(r+\pi /(2 n))^{n}$, где $r=|z|$. Имеем

$$
t_{1} \geqslant I_{1}=\int_{1}^{R(n)}\left(r+\frac{\pi}{2 n}\right)^{-n} d r, \quad t_{2} \geqslant I_{2}=\int_{R(n)}^{\infty}\left(r+\frac{\pi}{2 n}\right)^{-n} d r .
$$

Зафиксируем $R(n)$ так, чтобы вьполнялось равенство $2 I_{1}=I_{2}$, а именно,

$$
R(n)=\left(\frac{3}{2}\right)^{n-1}\left(1+\frac{\pi}{2 n}\right)-\frac{\pi}{2 n} .
$$


При этом

$$
2 I_{1}=I_{2}=\frac{2}{3(n-1)}\left(1+\frac{\pi}{2 n}\right)^{1-n}>T=\frac{1}{6 n} .
$$

Значит, $2 t_{1}>T$ и $t_{2}>T$. Следовательно, искомое $R(n)$ найдено.

2. Двойное отношение и уравнения, имеющие не больше трех периодических решений. Хорошо известно [4], что в случае уравнения Рикатти

$$
\dot{x}=x^{2}+a(t) x+b(t)
$$

отображение последования сохраняет двойное отношение (является проективным преобразованием прямой) и поэтому не может иметь более двух неподвижньх точек. Оказьвается, что двойное отношение полезно рассмотреть и в случае $n=3$.

ТЕорема 2. Рассмотрим дифференииальное уравнение вида

$$
\dot{x}=x^{3}+a(t) x^{2}+b(t) x+c(t)
$$

с гладкими коэффициентами $a(t), b(t), c(t)$. Обозначим через $\varphi(x, t)$ решение этого уравнения с начальным условием $\varphi(x, 0)=0$. Пусть $x_{1}, x_{2}, x_{3}, x_{4}$ - четыре произвольные точки на прямой, расположенные в порядке возрастания: $x_{1}<x_{2}<$ $x_{3}<x_{4}$. Пусть $S(\bar{x}, t)$ есть двойное отношение точек $\varphi\left(x_{1}, t\right), \varphi\left(x_{2}, t\right), \varphi\left(x_{3}, t\right)$, $\varphi\left(x_{4}, t\right):$

$$
S(\bar{x}, t)=\frac{\varphi\left(x_{1}, t\right)-\varphi\left(x_{2}, t\right)}{\varphi\left(x_{1}, t\right)-\varphi\left(x_{3}, t\right)} \cdot \frac{\varphi\left(x_{4}, t\right)-\varphi\left(x_{3}, t\right)}{\varphi\left(x_{4}, t\right)-\varphi\left(x_{2}, t\right)} .
$$

Тогда двойное отношение $S(\bar{x}, t)$ является возрастающей функиией времени.

ДокАЗАТЕЛЬСТВо. С точностью до бесконечно малых второго порядка имеем

$$
\begin{aligned}
S(\bar{x}, \Delta t)= & \frac{x_{1}-x_{2}+\Delta t\left(x_{1}^{3}-x_{2}^{3}+a(0)\left(x_{1}^{2}-x_{2}^{2}\right)+b(0)\left(x_{1}-x_{2}\right)\right)}{x_{1}-x_{3}+\Delta t\left(x_{1}^{3}-x_{3}^{3}+a(0)\left(x_{1}^{2}-x_{3}^{2}\right)+b(0)\left(x_{1}-x_{3}\right)\right)} \\
& \times \frac{x_{4}-x_{3}+\Delta t\left(x_{4}^{3}-x_{3}^{3}+a(0)\left(x_{4}^{2}-x_{3}^{2}\right)+b(0)\left(x_{4}-x_{3}\right)\right)}{x_{4}-x_{2}+\Delta t\left(x_{4}^{3}-x_{2}^{3}+a(0)\left(x_{4}^{2}-x_{2}^{2}\right)+b(0)\left(x_{4}-x_{2}\right)\right)} \\
= & \frac{x_{1}-x_{2}}{x_{1}-x_{3}} \cdot \frac{1+\Delta t\left(x_{1}^{2}+x_{1} x_{2}+x_{2}^{2}+a(0)\left(x_{1}+x_{2}\right)+b(0)\right)}{1+\Delta t\left(x_{1}^{2}+x_{1} x_{3}+x_{3}^{2}+a(0)\left(x_{1}+x_{3}\right)+b(0)\right)} \\
& \times \frac{x_{4}-x_{3}}{x_{4}-x_{2}} \cdot \frac{1+\Delta t\left(x_{4}^{2}+x_{4} x_{3}+x_{3}^{2}+a(0)\left(x_{4}+x_{3}\right)+b(0)\right)}{1+\Delta t\left(x_{4}^{2}+x_{4} x_{2}+x_{2}^{2}+a(0)\left(x_{4}+x_{2}\right)+b(0)\right)} \\
= & \frac{x_{1}-x_{2}}{x_{1}-x_{3}} \cdot \frac{x_{4}-x_{3}}{x_{4}-x_{2}} \cdot\left(1+\Delta t\left(x_{1}-x_{4}\right)\left(x_{2}-x_{3}\right)\right) .
\end{aligned}
$$

Учитьвая, что

$$
S(\bar{x}, 0)=\frac{x_{1}-x_{2}}{x_{1}-x_{3}} \cdot \frac{x_{4}-x_{3}}{x_{4}-x_{2}}
$$

и $x_{1}<x_{2}<x_{3}<x_{4}$, получаем, что знак инфинитезимального приращения совпадает со знаком $\left(x_{1}-x_{4}\right)\left(x_{2}-x_{3}\right)$, т.е. положителен. Ясно, что этот результата верен не только для $t=0$, но и вообще для любого $t$. Теорема доказана.

Обозначим через $\Phi(t)$ отображение $x \mapsto \varphi(x, t)$, где $\varphi(x, t)$ - решение уравнения (4) с начальньм условием $\varphi(x, 0)=0$. Из доказанной теоремы получаем 
СлЕДСТВИЕ 1. Отображсение $\Phi$ в любой момент времени имеет не более трех неподвижных точек.

Вычисления, аналогичные проделанньм при доказательстве теоремы, позволяют получить следующий более общий результат.

Теорема 3. Рассмотрим дифференииальное уравнение

$$
\dot{x}=f(x, t)
$$

с правой частью, обладающей тем свойством, что в каждый момент времени график функиии $f(x, t)$ пересекается $c$ любой параболой вида ах $x^{2}+b x+c$ не более чем в трех точках. В этом случае для любых четырех несовпадаюших решений двойное отношение $S(\bar{x}, t)$ является монотонной функиией времени.

Отметим, что условие, наложенное на функцию $f(x, t)$, по сути равносильно знакопостоянству ее третьей производной $\partial^{3} f / \partial^{3} x$.

ДоказАтельство. Рассмотрим четыре произвольные точки $x_{1}<x_{2}<x_{3}<x_{4}$ на прямой. Через точки $\left(x_{i}, f\left(x_{i}, t\right)\right), i=1,2,3$, проведем параболу $y(x)=a x^{2}+b x+c$. Из условий, наложенньх на функцию $f(x, t)$, следует, что разность $\omega=f\left(x_{4}, t\right)-y\left(x_{4}\right)$ будет знакопостоянной, т.е. ее знак не будет зависеть ни от времени, ни от конкретного значения $x_{4}$. Повторяя те же вычисления, что при доказательстве теоремы 2 , получаем, что с точностью до бесконечно малых второго порядка

$$
S(\bar{x}, t+\Delta t)=S(\bar{x}, t) \cdot\left(1+\Delta t \frac{\omega \cdot\left(x_{3}(t)-x_{2}(t)\right)}{\left(x_{4}(t)-x_{3}(t)\right)\left(x_{4}(t)-x_{2}(t)\right)}\right) .
$$

Отсюда следует, что знак инфинитезимального приращения функции $S(\bar{x}, t)$ постоянен и, значит, эта функция монотонна. Теорема доказана.

СлЕДСТВИЕ 2. В условиях теоремы 3 отображение $\Phi$ в любой момент времени имеет не более трех неподвиэных точек.

Отметим следующий частный случай следствия 2 , перекрьвающий следствие 1.

СлЕДСТВИЕ 3. Отображение последования дифференциального уравнения вида

$$
\dot{x}=x^{2 n+1}+a(t) x^{2}+b(t) x+c(t)
$$

не мохсет иметь более трех неподвижных точек.

3. Случай $n=4$ : контактная структура и поведение решений. Ограничимся дифференциальными уравнениями вида

$$
\dot{x}=\left(x^{2}-1\right)\left(x^{2}+a(t) x+b(t)\right)
$$

с периодически зависяшими от времени коэффициентами: $a(t+T)=a(t), b(t+T)=b(t)$. Оказывается, что поведение решений для всего этого класса уравнений регулируется следующей выделенной 1-формой:

$$
\alpha=\frac{x_{2}-x_{3}}{x_{1}^{2}-1} d x_{1}+\frac{x_{3}-x_{1}}{x_{2}^{2}-1} d x_{2}+\frac{x_{1}-x_{2}}{x_{3}^{2}-1} d x_{3} .
$$


ТЕОРема 4. Пусть $x_{1}(t), x_{2}(t), x_{3}(t)$ - три различных решения уравнения (5). Поставим им в соответствие кривую $\bar{x}(t)=\left(x_{1}(t), x_{2}(t), x_{3}(t)\right)$, лежсащую в $\mathbb{R}^{3}$. Тогда имеет место соотношение

$$
\alpha(\dot{\bar{x}}(t))=\left(x_{1}(t)-x_{2}(t)\right)\left(x_{2}(t)-x_{3}(t)\right)\left(x_{3}(t)-x_{1}(t)\right) .
$$

Верно и обратное утверждение. Пусть кривая $\bar{x}(t)=\left(x_{1}(t), x_{2}(t), x_{3}(t)\right)$ удовлетворяет соотношению $(6)$, причем $-1<x_{1}(t)<x_{2}(t)<x_{3}(t)<1$. Тогда найдутся функиии $a(t)$ и $b(t)$ такие, что функиии $x_{1}(t), x_{2}(t), x_{3}(t)$ являются решением уравнения (5).

Вот лемма, из которой следует первая часть теоремы.

Лемма 3. Пусть задана некоторая точка $\bar{x}=\left(x_{1}, x_{2}, x_{3}\right) \in \mathbb{R}_{3}$ и пара чисел $a, b$. Рассмотрим касательный вектор $\bar{y}=\left(y_{1}, y_{2}, y_{3}\right)$, приложсенный к точке $\bar{x}$ и имеющий координать

$$
\begin{gathered}
y_{1}=\left(x_{1}^{2}-1\right)\left(x_{1}^{2}+a x_{1}+b\right), \quad y_{2}=\left(x_{2}^{2}-1\right)\left(x_{2}^{2}+a x_{2}+b\right), \\
y_{3}=\left(x_{3}^{2}-1\right)\left(x_{3}^{2}+a x_{3}+b\right) .
\end{gathered}
$$

Тогда имеет место соотношение

$$
\alpha(\bar{y})=\left(x_{1}-x_{2}\right)\left(x_{2}-x_{3}\right)\left(x_{3}-x_{1}\right) .
$$

ДокАЗАТЕЛЬСтво. Имеем

$$
\begin{aligned}
\alpha(y)= & \frac{x_{2}-x_{3}}{x_{1}^{2}-1}\left(x_{1}^{2}-1\right)\left(x_{1}^{2}+a x_{1}+b\right)+\frac{x_{3}-x_{1}}{x_{2}^{2}-1}\left(x_{2}^{2}-1\right)\left(x_{2}^{2}+a x_{2}+b\right) \\
& +\frac{x_{1}-x_{2}}{x_{3}^{2}-1}\left(x_{3}^{2}-1\right)\left(x_{3}^{2}+a x_{3}+b\right) \\
= & \left(x_{2}-x_{3}\right) x_{1}^{2}+\left(x_{3}-x_{1}\right) x_{2}^{2}+\left(x_{1}-x_{2}\right) x_{3}^{2}=\left(x_{1}-x_{2}\right)\left(x_{2}-x_{3}\right)\left(x_{3}-x_{1}\right) .
\end{aligned}
$$

Лемма, а вместе с ней и первая часть теоремы доказаны.

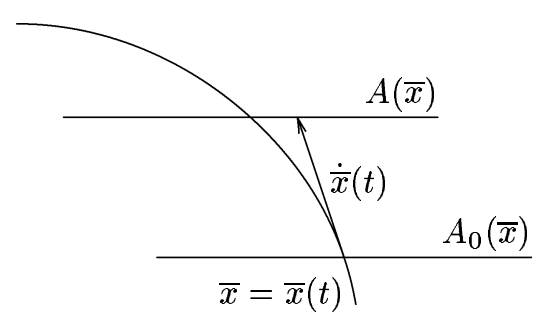

Рис. 2 
Соотношение (7) задает в каждом касательном пространстве $T_{\bar{x}} \mathbb{R}^{3}$ аффинную плоскость $A(\bar{x})$. Через $A_{0}(\bar{x})$ обозначим параллельную ей плоскость в $T_{\bar{x}} \mathbb{R}^{3}$, которая задается однородным уравнением $\alpha(\bar{y})=0$, а через $\mathscr{A}_{0}$ - поле, порожденное этими плоскостями. Геометрический смысл доказанной части теоремы заключается в том, что для вектора решений $\bar{x}(t)=\left(x_{1}(t), x_{2}(t), x_{3}(t)\right)$ конец вектора скорости $\dot{\bar{x}}(t)$ обязательно лежит в аффинной плоскости $A(\bar{x})$ (рис. 2$)$. Докажем оставшуюся часть теоремы.

ОКОНЧАНИЕ ДОКАЗАТЕЛЬСТВА ТЕОРЕМЫ. ПУсть крИвая $\bar{x}(t)=\left(x_{1}(t), x_{2}(t), x_{3}(t)\right)$ удовлетворяет соотношению (6). Рассмотрим систему уравнений

$$
\begin{aligned}
& \dot{x}_{1}(t)=\left(x_{1}^{2}(t)-1\right)\left(x_{1}^{2}(t)+a(t) x_{1}(t)+b(t)\right), \\
& \dot{x}_{2}(t)=\left(x_{2}^{2}(t)-1\right)\left(x_{2}^{2}(t)+a(t) x_{2}(t)+b(t)\right), \\
& \dot{x}_{3}(t)=\left(x_{3}^{2}(t)-1\right)\left(x_{3}^{2}(t)+a(t) x_{3}(t)+b(t)\right),
\end{aligned}
$$

где неизвестными являются функции $a(t)$ и $b(t)$. По условию левая часть этой системы определяет точку, лежащую в аффинной плоскости, задаваемой соотношением (7). С другой стороны, по лемме 3 при любьх $a(t)$ и $b(t)$ правая часть определяет точку, лежащую в той же самой аффинной плоскости. Ни в какой момент времени функции $x_{1}(t), x_{2}(t), x_{3}(t)$ не равны между собой. Отсюда следует, что, на самом деле, при подходящем выборе $a(t)$ и $b(t)$ может быть получена любая точка аффинной плоскости. Так что система имеет решение, при этом единственное. Теорема доказана.

Покажем, каким образом теорема 4 приводит к уравнениям с шестью циклами.

СЛЕДСТВИЕ 4. Пусть в области $-1<x_{1}<x_{2}<x_{3}<1$ задана замкнутая кривая $\gamma$, трансверсальная полю плоскостей $\mathscr{A}_{0}$, т.е. $\alpha(\dot{\gamma}) \neq 0$. Тогда существует параметризация кривой $\gamma(t)=\left(x_{1}(t), x_{2}(t), x_{3}(t)\right)$ и функиии $a(t)$ и $b(t)$ такие, что $x_{1}(t), x_{2}(t), x_{3}(t)$ являются решениями уравнения (4).

ДокАЗАТЕЛьство. В самом деле, так как в каждой точке $\bar{x}$ кривой $\gamma$ вектор $\dot{\gamma}$ трансверсален плоскости $A_{0}(\bar{x})$, параметр на кривой можно выбрать так, чтобы конец вектора $\dot{\gamma}$ лежал на параллельной аффинной плоскости $A(\bar{x})$. После этого остается применить вторую часть теоремы 4.

Сразу заметим, что если кривая, трансверсальная полю плоскостей $\mathscr{A}_{0}$, существует, то существует и уравнение с шестью периодическими решениями. Два из них очевидны: это $x=-1$ и $x=1$, три решения дает следствие 4 , а шестое получается из соображений четности (четность числа периодических решений любого уравнения вида (1) в общем случае равна четности степени $n)$.

Другой очевидньй факт заключается в том, что если поле плоскостей $\mathscr{A}_{0}$ интегрируемо, то не существует замкнутых кривых, трансверсальных этому полю. Поэтому необходимьм условием для существования уравнений вида (5) с шестью периодическими решениями является неинтегрируемость поля $\mathscr{A}_{0}$, которая доказьвается прямой проверкой условия $\alpha \wedge d \alpha \neq 0$ (см. [5]). Таким образом, поле задает контактную структуру [5]. В то же время всякую контактную структуру локальной заменой координат можно привести к стандартному виду $d z-x d y$ (теорема Дарбу [5]).

ПРЕДЛОЖЕНИЕ 1. В любой сколь угодно малой окрестности начала координат существует замкнутая кривая, трансверсальная стандартной контактной структуре. 
ДокаЗАТЕЛЬСтво. Рассмотрим кривую

$$
\gamma(t)=\left(\varepsilon \sin t, \varepsilon \cos t, \varepsilon^{2} \frac{\sin 2 t}{4}\right) .
$$

Имеем

$$
(d z-x d y)(\dot{\gamma})=\frac{\varepsilon^{2}}{2} .
$$

Предложение доказано. Вместе с ним доказано и существование уравнений вида (5) с шестью циклами.

Выражаю признательность Ю.С. Ильяшенко за постановку задач, за регулярные консультации и за всемерную поддержку. Также выражаю благодарность Д. А. Панову за многочисленные обсуждения этой работы.

\section{СПИСОК ЦИТИРОВАННОЙ ЛИТЕРАТУРЫ}

[1] Арнольд В. И., Ильяшенко Ю .С. Обыкновенные дифференциальные уравнения // Динамические системы. Итоги науки и техн. Соврем. пробл. матем. Фундамент. направления. Т. 1. М.: ВИНИТИ, 1985. С. 7-149.

[2] Плисс В.А. О числе периодических решений уравнения с полиномиальной правой частью // Докл. АН СССР. 1959. Т. 127. № 5. С. 965-968.

[3] Shahshahani S. Periodic solutions of polynomial first order differential equations // Nonlinear Anal. 1981. V. 5. № 2. P. 157-165.

[4] Степанов В.В.Курс дифференциальных уравнений. М.: ГИТТЛ, 1952.

[5] Арнольд В. И. Математические методы классической механики. М.: Наука, 1989. 